\title{
ARTÍCULO
}

\section{ANÁLISIS DE LOS CONSORCIOS TECNOLÓGICOS EMPRESARIALES EN CHILE**}

\author{
Roberto Álvarez \\ Carmen Contreras \\ José Luis Contreras \\ Intelis, Universidad de Chile
}

\begin{abstract}
Resumen: En este trabajo se analiza el funcionamiento de los consorcios tecnológicos en Chile. Utilizando datos de encuestas realizadas a empresas participantes, se encuentra que éstas atribuyen a estos programas un bajo impacto en su desempeño en materias de innovación tecnológica. No obstante, existe
\end{abstract}

Roberto Álvarez. Ph. D. in Management, University of California, Los Angeles (UCLA). Profesor Asociado de Economía e investigador del Centro Intelis, Universidad de Chile (Robalvar@fen.uchile.cl).

Carmen Contreras. MS in Economics, University of Nottingham; candidata a M. Phil. in Development Studies, University of Oxford. Investigadora del Centro Intelis, Universidad de Chile (Carmen.contreras@qeh.ox.ac.uk).

José Luis Contreras B. Magister en Economía, Universidad de Chile; MS en Economía, Universitat Pompeu Fabra. Investigador del Centro Intelis, Universidad de Chile (Jose.contreras@fen.uchile.cl).

* Este trabajo se basa en el estudio "Análisis Institucional de Instrumentos Consorcios Tecnológicos Empresariales (CTE) y Evaluación de Medio Término de Consorcios Tecnológicos" encargado por la Subsecretaría de Economía y el Fondo de Innovación Agraria del Gobierno de Chile. Agradecemos los valiosos comentarios y sugerencias de José Miguel Benavente, Gustavo Crespi y dos árbitros anónimos de Estudios Públicos, y también la eficiente asistencia de Rolando Campusano. Los autores agradecen el financiamiento del Programa Núcleo Milenio en Ciencias Sociales NS 100017 "Centro Intelis".

Estudios Públicos, 126 (otoño 2012). 
una mejor evaluación respecto a sus efectos sobre el acceso a conocimiento y desarrollo de nuevos proyectos tecnológicos en conjunto con otras empresas del consorcio. Dado que los resultados señalan un impacto relativamente bajo de estos programas, esto genera dudas de si efectivamente ellos están solucionando las fallas de mercado que justifican su subsidio por parte del Estado. Los resultados de este estudio sugieren que cambios en el diseño y la gestión de los consorcios y una definición más clara de sus objetivos y resultados esperados serían necesarios para mejorar su contribución al desempeño innovador de las empresas.

Palabras clave: Consorcios, innovación tecnológica, subsidios, asociatividad.

Recibido: marzo 2012; aceptado: mayo 2012.

\section{ANALYSIS OF TECHNOLOGICAL CONSORTIA IN CHILE}

Abstract: This paper analyzes the performance of Technological Consortia in Chile. Using information provided by a direct survey to participants, it is found that enterprises attribute to these programs a low impact on their performance in aspects related to technological innovation. A relatively higher evaluation is found in terms of improving access to technological knowledge and the development of new joint technological projects with other firms of the consortium. Given that the effects of these programs have been found to be relatively low, there are doubts that they have been contributing to solve the markets failures that justify their public funding. The results of this study suggest that changes in management and design, and a better definition of objectives and outcomes are needed to improve the contribution of consortia to the innovation performance of firms.

Keywords: consortia, technological innovation, public funding, associativity.

Received: March 2012; accepted: May 2012. 


\section{Introducción}

$\mathrm{L}$

os consorcios tecnológicos empresariales en Chile son de reciente funcionamiento. Se iniciaron el año 2004, con el primer concurso realizado bajo el marco del Programa Bicentenario de Ciencia y Tecnología $(\mathrm{PBCT})^{1}$. Este tipo de políticas, que se han implementado en diversos países desarrollados y en algunos en desarrollo, tiene como objetivo fundamental incrementar la colaboración entre distintos agentes relevantes, principalmente empresas y universidades. Esta colaboración se considera esencial para inducir un mayor gasto en investigación y desarrollo (I+D) y así acelerar el proceso innovador de las economías.

La justificación teórica de este tipo de instrumento es solucionar algunas de las fallas de mercado que afectan la innovación empresarial, mediante el incentivo a adoptar estrategias asociativas que ayuden a internalizar las externalidades de conocimiento, coordinar el uso de activos complementarios y compartir el riesgo tecnológico de las inversiones en innovación realizadas por agentes del sector privado.

Existen varios estudios en países desarrollados que han analizado los determinantes del éxito de estos instrumentos y también su efecto sobre el desempeño de las firmas participantes. Irwin y Klenow (1996) es uno de los primeros trabajos empíricos que analizan el efecto de los consorcios en el desempeño de las empresas. Para ello utilizan información de 14 empresas en el sector de semiconductores en los Estados Unidos y encuentran que la participación en consorcios redujo la inversión en I+D. Ello sería compatible con la idea de que, por efecto duplicación, la inversión individual sin consorcio es mayor a la que se obtiene dentro de un consorcio. Los resultados sobre variable de desempeño indican un efecto positivo sobre rentabilidad de las empresas, pero no así en la inversión y la productividad. Branstetter y Sakakibara (1998), por el contrario, encuentran que las empresas japonesas que participan en un consorcio incrementan su gasto en $\mathrm{I}+\mathrm{D}$ y la productividad de la investigación, medida como el número de patentes recibidas en los Estados Unidos. Estos efectos positivos serían

${ }^{1}$ El PBCT comenzó a funcionar el año 2003 con un préstamo del Banco Mundial y el Gobierno de Chile. Bajo la administración de la Comisión Nacional de Investigación Científica y Tecnológica (Conicyt) se estructuró en tres componentes: (i) mejoramiento del sistema de ciencia, tecnología e innovación, (ii) fortalecimiento de la base científica y (iii) fomento de la vinculación público-privada. 
explicados porque la participación en consorcios permiten aprovechar los spillovers de conocimiento entre sus miembros. En otro trabajo Branstetter y Sakakibara (2002) indagan sobre algunas características de los consorcios que favorecen su desempeño y encuentran que la proximidad tecnológica entre empresas, una medida de la potencial generación de spillovers de I+D y el énfasis en investigación básica tienen un efecto positivo sobre el número de patentes. Además, encuentran evidencia de que un mayor grado de competencia en el mercado del producto afecta negativamente los resultados del consorcio.

En el caso de los consorcios en Alemania, Czarnitzki y Fier (2003) analizan el efecto del financiamiento público en la productividad tecnológica de las empresas, medida por el número de patentes. Se encuentra que las empresas que se asocian son más propensas a patentar que las que no se asocian. Además, la evidencia indica que las empresas en consorcios financiados con recursos públicos tienen una propensión mayor a patentar que las que están asociadas a consorcio que no reciben financiamiento público.

Kaiser y Kuhn (2012) realizan una evaluación de impacto de los consorcios en Dinamarca. Utilizando un período de tiempo más extenso que el de los trabajos anteriores, analizan efectos contemporáneos y de más largo plazo. Se encuentra que la participación en un consorcio tiene un efecto positivo sobre la aplicación de patentes desde el primer hasta el tercer año de la participación en estos proyectos. Este impacto positivo se encuentra también en el empleo, pero no así en la productividad de las firmas. Estos efectos positivos son mayoritariamente explicados por firmas que tenían una mayor propensión a patentar aun antes de la participación en consorcios, pero no así por las firmas más grandes. Una implicancia de este trabajo es que los efectos positivos de la participación en consorcios, en general, toman tiempo en materializarse y dependen de ciertas características de las empresas.

Como lo sugiere esta revisión de la literatura, la mayoría de los estudios encuentran efectos positivos de la participación en consorcios sobre el desempeño de las firmas, pero existe poca evidencia respecto a factores que puedan explicar diferencias entre consorcios. Lo más parecido a lo que se realiza en este trabajo es el análisis empírico de Dyer et al. (2006) para los consorcios en Estados Unidos. Estos autores incluyen una larga lista de factores que pueden afectar el desempeño de los consorcios. Entre ellos, su diseño, que tiene que ver con la estructura del consorcio y los atributos de las firmas participantes, y su 
administración, que se relaciona con la forma en que se organizan y asignan los recursos dentro del consorcio. Sus resultados indican que el número de participantes y la presencia de competidores no ejercen una influencia importante sobre el desempeño de las empresas, medido por la evaluación de los participantes y si la empresa aplicó a patentes o no como resultado de su participación en el consorcio. Entre los factores que mejoran el desempeño destacan la existencia de acuerdos efectivos de governance al interior del consorcio, una frecuencia alta de comunicaciones y el establecer objetivos ambiciosos ${ }^{2}$.

En el caso de los países en vías de desarrollo, la evidencia acerca de los potenciales efectos de los consorcios es escasa. Tampoco existen trabajos que analicen si las características de estos consorcios, ya sea su diseño o los mecanismos de gestión interna, están asociadas a mejores indicadores de desempeño. Salvo algunos estudios de casos, como el realizado en México por Casalet y Stezano (2006), existe un alto grado de desinformación respecto a cómo estos programas han estado funcionando y si efectivamente pueden constituir una herramienta eficiente y efectiva para mejorar el desempeño innovador de las economías. Ello a pesar de que, en algunos casos, los consorcios llevan varios años de funcionamiento y los recursos públicos involucrados han sido de una magnitud importante.

En el caso de Chile, no existen análisis relativos al desempeño de los consorcios y sus efectos sobre las empresas - y otras entidades- participantes ${ }^{3}$. Este trabajo es un primer intento de estudiar el funcionamiento de los consorcios tecnológicos empresariales en Chile y analizar si existen aspectos de su diseño y funcionamiento que puedan ser mejorados para permitir un efecto mayor sobre las actividades de innovación. Aun cuando, dada la información con que se cuenta, no es posible realizar una evaluación formal de impacto, el análisis efectuado permite entregar elementos importantes para una evaluación preliminar del desempeño de este tipo de instrumentos.

${ }^{2}$ Un trabajo relacionado es el de Acosta y Modrego (2001) que estudian los determinantes de la asignación de financiamiento público a los consorcios en España.

${ }^{3}$ La excepción es el trabajo realizado por el Centro Intelis en el marco de un proyecto sobre consorcios en América Latina financiado por el BID y el IDRC (Álvarez et al. 2012). Sin embargo, el bajo número de respuestas obtenidas por entidades chilenas no permite hacerlo comparable con este estudio. 
Este trabajo se estructura de la siguiente manera. En la sección 2 se describen el instrumento y los consorcios bajo análisis, así como la fuente de los datos. En la sección 3, se muestran los principales resultados, basados en encuestas realizadas a empresas participantes. La sección 4 presenta un análisis econométrico de los factores determinantes del desempeño de los consorcios. Finalmente, en la sección 5, se resumen los principales resultados y se discuten algunas implicancias de política.

\section{Descripción del instrumento y los consorcios bajo estudio}

Durante las últimas décadas se han desarrollado varios instrumentos para mejorar el desempeño en la economía chilena. Entre éstos destacan los denominados consorcios tecnológicos empresariales de investigación. Esta iniciativa se enmarca en el Programa Bicentenario de Ciencia y Tecnología (PBCT), impulsado por la Comisión Nacional de Investigación Científica y Tecnológica (Conicyt), con el aporte de fondos del Banco Mundial, la Corporación de Fomento de la Producción (Corfo) y la Fundación para la Innovación Agraria (FIA). Los recursos involucrados en este tipo de iniciativas son de una magnitud importante. En el caso de los once consorcios analizados en este estudio, el aporte público fue de aproximadamente 25 mil millones de pesos, lo que da un promedio de 2.200 millones de pesos por consorcio 4 .

Este instrumento busca que los resultados obtenidos de la investigación sean posibles de empaquetar en un producto o servicio para posteriormente comercializarlo y/o difundirlo. Para ello, se fomenta la formación de empresas con las capacidades de tener como principal giro de negocio el desarrollar investigación de punta que esté orientada a satisfacer las necesidades que los sectores productivos puedan demandar.

Se considera pertinente en la estrategia de las empresas participantes la obtención de patentes de los resultados, la venta de licencias de las investigaciones y constituir una plataforma de spin-off para nuevas empresas, que tengan como activo inicial el resultado de las investigaciones del mismo consorcio. Para el cumplimiento de estos objetivos,

${ }^{4}$ Los montos corresponden al total del proyecto y están expresados en moneda del 2011. 
se requiere la asociación entre dos tipos de agentes: las empresas o representantes del sector productivo y las instituciones de investigación, principalmente universidades y/o centros de investigación. Con ello se pretende generar una instancia para que el mundo académico y el productivo interactúen para lograr los objetivos antes mencionados.

El diseño del instrumento está basado en el modelo de consorcio aplicado en Australia, denominado Programa de Centros de Investigación Cooperativa (CRC Programme), el cual ha estado operando desde hace veinte años aproximadamente. La experiencia en el caso australiano presta particular atención al tema de la vinculación entre el mundo académico y el productivo. Con este tipo de instancias se pretende converger gradualmente a una situación en la cual los investigadores entiendan las necesidades que el mundo productivo necesita desarrollar y sus plazos asociados y, por otro lado, el sector productivo comprenda la relevancia que tiene el proceso de investigación para su estrategia de negocios.

En Chile, la primera convocatoria de este instrumento fue realizada en 2004 y se seleccionaron nueve proyectos, creándose los primeros consorcios tecnológicos empresariales de investigación. El 2005 se realizó una segunda convocatoria. En ambos casos, el objetivo fundamental fue potenciar la asociatividad en el desarrollo de proyectos tecnológicos, co-financiando las actividades de I+D por un período de hasta cinco años ${ }^{5}$. Se esperaba que los consorcios cumplieran con las siguientes condiciones: (i) que el objetivo a alcanzar justificara la aplicación de este instrumento, considerando como condición mínima la necesidad de asociarse entre distintas entidades empresariales y entidades asociadas a la investigación, (ii) que la vinculación justificara avanzar en un esfuerzo sostenido en $\mathrm{I}+\mathrm{D}$ en base al consorcio, (iii) que el proyecto contribuyera significativamente al fortalecimiento de industrias existentes y la creación de negocios en áreas emergentes, (iv) que el proyecto necesitase del financiamiento público para ser realizado y (v) que existiese coherencia entre los recursos solicitados, y también entre dichos montos y los productos y resultados que se esperaban del desarrollo de los mismos.

${ }^{5}$ En la convocatoria de 2005, por ejemplo, se menciona explícitamente que se busca fomentar las iniciativas de asociatividad entre los distintos actores de la cadena productiva. 
En esta convocatoria se establecieron tres tipos de consorcios tecnológicos:

Consorcios universidad-empresa: Son iniciativas destinadas a ejecutar programas de I+D con aplicación productiva, de comercialización de los resultados de investigación y creación de capacidades permanentes de $\mathrm{I}+\mathrm{D}$, conjuntamente entre universidades, entidades de investigación y empresas. Estos consorcios deben contar al menos entre sus entidades principales con una universidad o institución de investigación nacional y una empresa nacional o una entidad empresarial representativa. Asimismo, deben considerar la participación de dos empresas adicionales (nacionales o extranjeras), ya sea como entidades principales o asociadas. Estos consorcios universidad-empresa deben ser presentados exclusivamente en Conicyt.

Consorcios empresariales: Son aquellos consorcios que hayan constituido o prevén la constitución de una persona jurídica cuya finalidad principal sea aprovechar una oportunidad a través de la creación de un negocio de base tecnológica con un fuerte componente de innovación tecnológica y cuyos socios correspondan, a lo menos, a dos empresas más una entidad tecnológica ${ }^{6}$. Los proyectos de consorcios empresariales tienen por objeto facilitar: (i) el desarrollo de proyectos de innovación con clara orientación al mercado, (ii) la incorporación temprana de socios tecnológicos y agentes empresariales a los proyectos, (iii) la concreción de alianzas entre empresas y entidades tecnológicas, nacionales y/o extranjeras, y (iv) la formulación de estrategias de negocios a partir de los productos y resultados innovadores que se obtienen de la ejecución de proyectos de desarrollo e innovación tecnológica. Estos consorcios deben ser presentados exclusivamente en Corfo.

Consorcios sectoriales agrarios: Son iniciativas que generen o prevean la constitución de una nueva persona jurídica cuya finalidad principal sea fortalecer la competitividad del sector agrícola, pecuario, forestal o dulceacuícola, mediante la investigación aplicada y desarro1lo. Las iniciativas deben ser ejecutadas integralmente por asociaciones de empresas y organizaciones relevantes de la cadena productiva y enti-

${ }^{6}$ Esta última podrá participar del consorcio en calidad de socio de la nueva persona jurídica que se cree o bien vincularse a ella mediante un convenio de co-ejecución o subcontrato. 
dades tecnológicas o universidades con capacidades en la materia. Estos consorcios deben ser presentados exclusivamente en la FIA (Fundación para la Innovación Agraria).

Los consorcios analizados en este trabajo se presentan en la Tabla $\mathrm{N}^{\circ} 1$ junto con la agencia pública encargada de su funcionamiento. El criterio de selección provino de las agencias y consistió en analizar prioritariamente los consorcios asociados a los primeros concursos de modo de contar con un razonable período de funcionamiento para analizar sus resultados. En particular todos los consorcios de la Tabla $\mathrm{N}^{\circ} 1$ provienen del primer concurso de 2004, con la excepción del Consorcio Ovino, Consorcio Apícola y Consorcio de la Papa los cuales están asociados al concurso de 2005.

TABLA N ${ }^{\circ}$ 1: $\quad$ CONSORCIOS ANALIZADOS

\begin{tabular}{ll}
\hline Agencia pública & Nombre del consorcio \\
\hline $\begin{array}{l}\text { Innova Chile } \\
\text { Innova Chile }\end{array}$ & $\begin{array}{l}\text { Consorcio Tecnológico Empresarial para la Vid y el Vino } \\
\text { Cnnova Chile }\end{array}$ \\
Conicyt & $\begin{array}{l}\text { Consorcio Genómica Forestal } \\
\text { Biofrutales (Innovación biotecnológica en la producción de nuevas } \\
\text { variedades de vides y frutales de carozo) }\end{array}$ \\
Conicyt & Naturales (Desarrollo de tecnologías innovadoras y competitivas para \\
& $\begin{array}{l}\text { la obtención de productos de alto valor agregado a partir de corrientes } \\
\text { residuales de la industria nacional forestal, pesquera y vitivinícola) }\end{array}$ \\
Conicyt & Consorcio de Tecnología e Innovación para la Salud (CTI-Salud) \\
Conicyt & Consorcio de Aeronáutica \\
FIA & Consorcio Tecnológico de la Leche \\
FIA & Consorcio Tecnológico de la Fruta \\
FIA & Consorcio Ovino \\
FIA & Consorcio Tecnológico Apícola para el Mercado Global \\
FIA & Consorcio Papa
\end{tabular}

Fuente: Elaboración propia basada en información proporcionada por las agencias respectivas.

Las bases del concurso 2005 entregan antecedentes sobre los criterios de evaluación de los proyectos presentados. Los seis criterios generales mencionados son los siguientes:

- Que los impactos producidos a partir del proyecto postulante contribuyan al crecimiento económico de Chile. 
- Que los impactos producidos a partir del proyecto postulante contribuyan a la creación de capacidades permanentes en los ámbitos de investigación, desarrollo e innovación del país.

- Que los modelos de adopción y transferencia de los resultados del proyecto permitan alcanzar los impactos comprometidos.

- Que los participantes del consorcio, la modalidad de colaboración presentada y el grado de compromiso permitan alcanzar los resultados esperados.

- El grado de compromiso económico de las entidades participantes y la rentabilidad sobre el financiamiento solicitado.

- El impacto tecnológico y la calidad de formulación técnica.

Para algunos de ellos se detalla más explícitamente una serie de criterios específicos. En el caso del primer criterio se menciona que se tendrá en consideración, entre otros, el desarrollo de nuevas industrias o el fortalecimiento de las actuales y el desarrollo de conglomerados regionales (clusters). En el segundo criterio correspondiente a la creación de capacidades permanentes, se mencionan como criterios de evaluación algunos aspectos más medibles como la instalación de nuevo equipamiento en universidades y/o empresas y la formación e inserción de recursos humanos altamente calificados, especialmente a nivel de postgrado. En el caso de los otros criterios no existe una definición de aspectos específicos.

Como se puede inferir de estos antecedentes, la evaluación ex ante de los consorcios consideraba una amplia gama de criterios. Algunos con un grado de generalidad tan amplia que hace difícil hacer un vínculo con medidas específicas de desempeño y/o resultados. Por ello, el análisis ex post, como el realizado en este trabajo, requiere primero seleccionar un grupo amplio de indicadores que están relativamente relacionados con los objetivos planteados.

En el caso particular de este trabajo, las medidas de desempeño utilizadas fueron discutidas previamente con la Subsecretaría de Economía y las agencias respectivas. Las 16 medidas utilizadas reflejan la necesidad de capturar potenciales impactos en materia de innovación propiamente tal. Dichas medidas fueron las siguientes: introducción de productos nuevos, introducción de procesos productivos nuevos, introducción de diseños industriales nuevos, mejoramiento de productos ya existentes, mejoramiento de procesos productivos ya existentes, mejoramiento de diseños industriales ya existentes, patentes de propiedad in- 
telectual solicitadas, aumento de las ventas, reducción de costos de producción, reducción del riesgo de la innovación, mejoramiento de acceso a conocimiento tecnológico, mejoramiento de acceso a conocimiento en otras áreas, mejoramiento en su desempeño exportador, obtención de certificaciones nacionales y/o internacionales, desarrollo de nuevos proyectos tecnológicos con socios del consorcio y desarrollo de nuevos proyectos comerciales con socios del consorcio.

\section{Fuente de los datos}

Dada la ausencia de información sistemática ${ }^{7}$ sobre el desempeño y características de los consorcios y las entidades participantes, este estudio se basa en información primaria proveniente de encuestas a las empresas participantes ${ }^{8}$. El cuestionario fue diseñado siguiendo fundamentalmente la literatura previa en el tema, especialmente Dyer et al. (2006), y está compuesta de varias secciones que buscan como objetivo proveer información respecto a (i) características de las empresas, (ii) origen y formalización del consorcio, (iii) diseño del consorcio, (iv) localización de los participantes, (v) características de la gestión, (vi) medidas de desempeño, (vii) identificación de obstáculos, (viii) proyectos y modelo de negocios y (ix) vinculación con agencias gubernamentales ${ }^{9}$.

En la Tabla $\mathrm{N}^{\circ} 2$ se resume el número de encuestas realizadas ${ }^{10}$. El marco muestral para la aplicación del cuestionario son todas las empresas y asociaciones gremiales participantes en los consorcios. Se obtuvo un total de 65 respuestas, que representan una tasa de respuesta de $90,3 \%$, dado un universo de 72 potenciales empresas a encuestar.

${ }^{7} \mathrm{Si}$ bien las agencias cuentan con información que puede ser de utilidad, como por ejemplo informes técnicos y financieros elaborados por los consorcios, restricciones de confidencialidad impidieron un análisis de este tipo de información.

${ }^{8}$ El trabajo de campo se complementó con entrevistas estructuradas a los gerentes de los consorcios y a los investigadores de universidades y/o institutos públicos participantes.

${ }^{9}$ En el Anexo se presenta el cuestionario completo.

${ }^{10}$ Aunque hacemos mención a empresas como la unidad encuestada, en algunos casos esto corresponde a asociaciones gremiales. En todos los casos corresponde a un entrevistado por empresa o asociación gremial. 


\begin{tabular}{lrc}
\hline Observaciones por agencia y consorcio & $\mathrm{N}$ & \% del total \\
\hline Innova Chile & & \\
CTE para la Vid y el Vino & 12 & 18,5 \\
Centro Cooperativo Desarrollo Vitivinícola & 1 & 1,5 \\
$\quad$ Genómica Forestal & 2 & 3,1 \\
Conicyt & 6 & \\
$\quad$ Biofrutales & 3 & 9,2 \\
Naturalis & 3 & 4,6 \\
CTI-Salud & & 4,6 \\
FIA & 7 & 10,8 \\
Consorcio Tecnológico de la Leche & 1 & 1,5 \\
Consorcio Tecnológico de la Fruta & 5 & 7,7 \\
Consorcio Ovino & 14 & 16,5 \\
Consorcio Papa & 11 & 100,0 \\
Consorcio Tecnológico Apícola & 65 & \\
Total & & \\
\end{tabular}

Fuente: Elaboración propia.

\section{Evaluación de desempeño}

Uno de los elementos relevantes en la evaluación de un instrumento es determinar los aspectos a ser evaluados. Naturalmente éstos deben estar definidos por los objetivos explícitos del instrumento bajo estudio. En este caso, los objetivos planteados por las convocatorias a los proyectos no definen resultados específicos que puedan ser sujetos de evaluación. Este trabajo, por lo tanto, usa una amplia gama de indicadores que la literatura sugiere pueden ser relevantes para determinar los efectos esperados de la participación en un consorcio. En primer lugar, se consideran indicadores relativos a resultados concretos en materia de innovación tecnológica, como es la introducción de productos, mejoramiento de procesos y la solicitud de patentes de propiedad intelectual. En segundo lugar, se consideran variables que intentan capturar si la participación en consorcios se ha traducido en mejoramiento del desempeño general de las empresas, como es el aumento de las ventas, la reducción de costos y el aumento de las exportaciones. Finalmente, se consideran indicadores que intentan medir si los consorcios han teni- 
do efectos en materia de acceso a conocimiento y reducción en el riesgo de la innovación.

La encuesta indaga sobre los potenciales efectos de la participación en el consorcio, y se pregunta - en una escala de 0 a 4 - en qué medida el consorcio ha contribuido hasta ahora a mejorar el desempeño de las empresas en 16 aspectos ${ }^{11}$. Los resultados de estas variables de desempeño se muestran en la Tabla $\mathrm{N}^{\circ} 3$. En general, la percepción de las empresas participantes es que su participación en los programas ha contribuido a su desempeño en una intensidad menor o igual a "moderadamente baja" (la máxima calificación promedio es 2,2). Ello es consistente con la evidencia recogida en varias de las entrevistas con gerentes de consorcios y agentes de organismos públicos, ya que una parte considerable de los consorcios analizados tienen objetivos no directamente relacionados con el mejoramiento en las capacidades y actividades innovadoras, sino que a mejorar la competitividad y en algunos casos la asociatividad de ciertos sectores.

TABLA N ${ }^{\circ}$ 3: $\quad$ CONTRIBUCIÓN DE LOS CONSORCIOS AL MEJORAMIENTO DE DESEMPEÑO

\begin{tabular}{lcccc}
\hline & Promedio & Desv. estándar & Mínimo & Máximo \\
\hline Productos nuevos & 1,4 & 1,3 & 0 & 4 \\
Procesos nuevos & 1,4 & 1,4 & 0 & 4 \\
Diseños industriales nuevos & 0,8 & 1,0 & 0 & 3 \\
Mejora productos existentes & 1,6 & 1,4 & 0 & 4 \\
Mejora procesos existentes & 1,4 & 1,2 & 0 & 4 \\
Mejora diseños industriales existentes & 0,8 & 1,0 & 0 & 4 \\
Patentes & 0,7 & 1,2 & 0 & 4 \\
Aumento ventas & 1,0 & 1,3 & 0 & 4 \\
Reducción costos & 1,0 & 1,1 & 0 & 4 \\
Reducción riesgo innovación & 1,4 & 1,2 & 0 & 4 \\
Acceso conocimiento & 2,3 & 1,3 & 0 & 4 \\
Acceso conocimiento otras áreas & 1,9 & 1,2 & 0 & 4 \\
Desempeño exportador & 0,8 & 1,2 & 0 & 4 \\
Certificaciones & 0,9 & 1,2 & 0 & 4 \\
Nuevos proyectos consorcio & 1,7 & 1,4 & 0 & 4 \\
Nuevos proyectos otras firmas & 1,4 & 1,3 & 0 & 4 \\
\hline
\end{tabular}

${ }^{11}$ La escala de respuesta utilizada para las preguntas de evaluación y obstáculos del desempeño de los consorcios es la siguiente: (0) Nula, (1) Baja, (2) Moderadamente baja, (3) Moderadamente alta, (4) Alta. 
En términos de los aspectos individuales, los resultados de la encuesta revelan que las tres principales contribuciones de los consorcios han sido el mejoramiento de acceso a conocimiento tecnológico, el mejoramiento de acceso a conocimiento en otras áreas (marketing, mercados internacionales, personal capacitado, etc.) y el desarrollo de proyectos tecnológicos conjuntos con empresas del consorcio. La contribución menos relevante de los consorcios está asociada a la solicitud de patentes de propiedad intelectual.

La agrupación de los indicadores en los tres grupos definidos anteriormente, innovación, desempeño general y acceso a conocimiento, indican que la evaluación de las empresas es más alta en indicadores de accesos a conocimiento que respecto a resultados más concretos en innovación y desempeño general (Tabla $\left.\mathrm{N}^{\circ} 4\right)$.

TABLA N ${ }^{\circ}$ 4: $\quad$ CONTRIBUCIÓN AL MEJORAMIENTO DE DESEMPEÑO POR GRUPO DE INDICADORES

Indicadores

Promedio

Desv. estándar

Mínimo

Máximo

Innovación

Desempeño general

Conocimiento

$\begin{array}{ll}1,4 & 1,2 \\ 1,0 & 1,1 \\ 1,8 & 1,0\end{array}$

0
0
0

4

4

4

En relación a los efectos esperados a futuro de la participación en el consorcio, la calificación tiende a ser generalmente más alta que la situación actual. Se encuentra que varios aspectos son evaluados con valores cercanos o iguales a "moderadamente alto". Como se aprecia en la Tabla $\mathrm{N}^{\circ} 5$, destaca el desarrollo de nuevos proyectos con miembros del consorcio, y, en comparación con los resultados obtenidos hasta ahora, se incrementa bastante la calificación en términos de aspectos más fuertemente ligados a la innovación tecnológica, como son la introducción de productos nuevos y el mejoramiento de productos existentes.

En términos de los tres principales grupos de indicadores, los efectos esperados en acceso a conocimiento siguen prevaleciendo sobre los resultados en innovación y desempeño general (Tabla $\mathrm{N}^{\circ} 6$ ). Esto sugiere que, aun cuando los efectos esperados en resultados para las empresas mejoran respecto a la situación actual, se sigue considerando 


\begin{tabular}{lcccc}
\hline & Promedio & Desv. estándar & Mínimo & Máximo \\
& & & & \\
Productos nuevos & 3,0 & 1,1 & 0 & 4 \\
Procesos nuevos & 2,8 & 1,2 & 0 & 4 \\
Diseños industriales nuevos & 2,0 & 1,2 & 0 & 4 \\
Mejora productos existentes & 3,0 & 1,1 & 0 & 4 \\
Mejora procesos existentes & 2,6 & 1,3 & 0 & 4 \\
Mejora diseños industriales existentes & 2,1 & 1,3 & 0 & 4 \\
Patentes & 2,0 & 1,4 & 0 & 4 \\
Aumento ventas & 2,6 & 1,4 & 0 & 4 \\
Reducción costos & 2,4 & 1,4 & 0 & 4 \\
Reducción riesgo innovación & 2,5 & 1,4 & 0 & 4 \\
Acceso conocimiento & 2,3 & 1,3 & 0 & 4 \\
Acceso conocimiento otras áreas & 3,0 & 1,0 & 0 & 4 \\
Desempeño exportador & 2,6 & 1,2 & 0 & 4 \\
Certificaciones & 2,5 & 1,3 & 0 & 4 \\
Nuevos proyectos consorcio & 3,1 & 1,0 & 0 & 4 \\
Nuevos proyectos otras firmas & 2,9 & 1,1 & 0 & 4 \\
& & & & \\
\hline
\end{tabular}

TABLA N ${ }^{\circ}$ :

CONTRIBUCIÓN ESPERADA AL DESEMPEÑO POR GRUPO DE INDICADORES

\begin{tabular}{lcccc}
\hline Indicadores & Promedio & Desv. estándar & Mínimo & Máximo \\
\hline Innovación & 2,3 & 0,9 & 0 & 3,8 \\
Desempeño general & 2,6 & 1,1 & 0 & 4 \\
Conocimiento & 3,0 & 0,8 & 0 & 4 \\
& & & & \\
\hline
\end{tabular}

que los principales efectos asociados a los consorcios es el mejoramiento en el acceso a conocimiento. Este resultado hace surgir interrogantes de si este instrumento es el adecuado para abordar los problemas de acceso a conocimiento de las empresas o existen alternativas más eficientes de lograr este objetivo.

Para explorar diferencias entre consorcios, se agrupan aquellos que operan bajo las tres instituciones públicas encargadas de su funcionamiento ${ }^{12}$. Los resultados para el promedio general y para los tres gru-

12 Dado que para algunos consorcios existe un bajo número de observaciones no se hacen comparaciones entre ellos. 
TABLA N ${ }^{\circ} 7: \quad$ CONTRIBUCIÓN AL DESEMPEÑO POR INSTITUCIÓN

\begin{tabular}{lcccccr}
\hline & $(1)$ & $(2)$ & $(3)$ & \multicolumn{2}{c}{ Test de igualdad de medias (p-value $)$} \\
\cline { 5 - 7 } & FIA & Conicyt & Innova & $(1)=(2)$ & $(1)=(2)$ & $(1)=(3)$ \\
\hline Total & 1,4 & 1,2 & 1,8 & 0,57 & 0,13 & 0,21 \\
Innovación & 1,4 & 1,0 & 1,7 & 0,30 & 0,10 & 0,36 \\
Desempeño general & 1,1 & 0,7 & 0,9 & 0,32 & 0,55 & 0,64 \\
Conocimiento & 1,6 & 1,8 & 2,2 & 0,53 & 0,31 & 0,06 \\
\hline
\end{tabular}

pos de indicadores se muestran en la Tabla $\mathrm{N}^{\circ}$ 7. La evidencia muestra, consistente con la evaluación general, que las empresas atribuyen un bajo impacto en su desempeño a la participación en los consorcios. Aun cuando, en general, las empresas bajo Innova Chile (Corfo) le atribuyen a su participación en el consorcio mayores efectos que el resto, las diferencias no son generalmente estadísticamente significativas, salvo para el caso de acceso a conocimiento.

Los resultados anteriores indican que las empresas, en general, atribuyen una baja importancia a los consorcios en su desempeño. La encuesta también indaga respecto a qué factores podrán estar afectando el funcionamiento de este instrumento y no generando los resultados esperados. En la Tabla $\mathrm{N}^{\circ} 8$ se muestran, por orden de importancia, los principales obstáculos identificados por las empresas. Se destaca que

TABLA N ${ }^{\circ}$ 8: $\quad$ OBSTÁCULOS AL DESEMPEÑO DE LOS CONSORCIOS

Obstáculo

Promedio

Diferentes objetivos entre empresas y universidades y/o centros de investigación

Diferentes enfoques de investigación entre empresas y universidades y/o centros

Falta de recursos humanos calificados en el país

Diferentes enfoques de investigación entre empresas

Diferentes objetivos entre empresas

Falta de recursos humanos calificados entre los miembros

Falta de recursos financieros 
ninguno de los obstáculos es indicado como uno de importancia relativamente alta. De hecho, el máximo promedio es 2, equivalente a una calificación moderadamente baja, y que corresponde a las diferencias de objetivos entre empresas y universidades y/o centros de investigación. En cambio, el aspecto de menor importancia relativa para una mejor contribución de los consorcios es la insuficiente capacidad de las empresas para adaptar y/o absorber los desarrollos tecnológicos generados por el consorcio. Además, el alto riesgo tecnológico de los proyectos no parece ser un obstáculo importante.

\section{Variables asociadas al desempeño de los consorcios}

En esta sección, basados en estudios previos, se utiliza un análisis econométrico para identificar aquellos factores más asociados al desempeño de los consorcios. Considérese una variable desempeño $Y_{i c}$ que es específica a una empresa $i$ que pertenece al consorcio $c$. En este caso, la variable corresponde a la percepción del encuestado respecto a si su participación en el consorcio ha contribuido a mejorar su desempeño en un aspecto determinado, por ejemplo, en términos de introducción de productos nuevos o patentes de propiedad intelectual solicitadas. Para ello, se utiliza la respuesta del encuestado en una escala de cinco alternativas: 0: nulo, 1: baja, 2: moderadamente baja, 3: moderadamente alta y 4: alta. El promedio de cada uno de los 16 aspectos evaluados, se toma como el indicador de desempeño general del consorcio percibido por la empresa.

Para estudiar los factores que afectan la evaluación del consorcio, utilizando un modelo de regresión lineal, se estima la siguiente ecuación:

$$
Y_{i c}=\alpha+\beta X_{c}+\delta Z_{i}+\varepsilon_{i c}
$$

Donde $Y_{i c}$ es una medida subjetiva de desempeño reportada por la empresa $i$ para el consorcio $c$. Esta medida depende, entre otras cosas, de ciertas características del diseño y la gestión de consorcio (número de miembros, frecuencia de comunicación entre las partes, localización de las entidades, etc.), que podemos denotar por $X_{i c}$, y de características de las empresas participantes (tamaño, gasto en $\mathrm{I}+\mathrm{D}$, etc.) que podemos 
denotar por $Z_{i}$. Para explorar los factores que están relacionados con diferencias en desempeño de los consorcios, se consideran tres principales grupos de variables: (i) diseño, (ii) gestión y (iii) características de las empresas participantes.

En el caso del diseño, se utiliza una variable categórica si la empresa indica que el consorcio cuenta con empresas de la misma industria que compiten directamente con ella (competidores). De acuerdo a la literatura previa, se espera que la presencia de competidores afecte negativamente el desempeño de los consorcios, ello por cuanto una mayor competencia incrementa los riesgos que las empresas reduzcan sus esfuerzos de colaboración y compartan conocimiento con otras empresas (Dyer et al., 2006; Branstetter y Sakakibara, 2002).

Se incluye también una variable que captura la distancia entre las empresas y entre las empresas y el gerente o coordinador del consorcio (Distancia). Esta variable toma valores de 1 a 4, con un mayor valor indicando más distancia. Se espera que la mayor distancia entre miembros afecte negativamente el desempeño de los consorcios, ya que la proximidad geográfica influye de manera importante en facilitar la interacción y el flujo de conocimientos entre firmas de un consorcio. Mientras más cercanas están, mayores son las posibilidades de interacción entre las firmas y menores los costos de comunicación (Saxenian, 1994; Almeida y Kogut, 1999).

También, se incluye una medida de si la empresa ha colaborado previamente en otros proyectos tecnológicos con las empresas del consorcio (Colaboración previa). Aquellas firmas que ya han participado en alianzas y asociaciones desarrollan "capacidades relacionales" (relational capabilities), las que podrían conducir a un mejor funcionamiento de los consorcios. Sin embargo, este efecto puede ser positivo sólo en el caso que exista experiencia previa de cooperación con un socio específico que forma parte del consorcio (Dyer et al., 2006). Relacionado también con los atributos está la importancia de la capacidad de las firmas para internalizar y generar $\mathrm{I}+\mathrm{D}$, para lo cual podría ser necesario contar con una adecuada capacidad de absorción ${ }^{13}$.

${ }^{13}$ No existe una única manera de capturar este efecto, lo más común es usar alguna medida de capital humano de la firma o su gasto relativo en I+D. En nuestro caso, como se discute más adelante, usamos como variable explicativa si la empresa declara tener un departamento de $\mathrm{I}+\mathrm{D}$. 
En términos de gestión, se incluyen tres variables categóricas que capturan la frecuencia de comunicación entre las empresas correspondiente a mensual, trimestral y anual. La categoría base excluida es la frecuencia semanal. En este caso, se espera que una menor frecuencia de comunicaciones afecte negativamente el desempeño. El fundamento de esta relación es que comunicaciones más frecuentes facilitan el intercambio de conocimiento entre los socios del consorcio, contribuyendo así a un mejor desempeño.

También se considera el rol de las reglas explícitas en la gestión del consorcio. En este caso, se considera si el consorcio tiene reglas para la salida o entrada de firmas y para el uso de resultados. Esta variable toma valores entre 0 , si no hay ninguna de ambas reglas, hasta $2 \mathrm{si}$ existen ambas (Reglas). En este caso se espera una relación positiva con el desempeño del consorcio. La justificación de incluir esta variable es tratar de analizar la hipótesis de que un mayor grado de satisfacción con los arreglos contractuales entre los socios, el que puede estar asociado a la existencia de reglas explícitas, mejora el desempeño del consorcio (Dyer et al., 2006).

Finalmente, como variables de control para las características de las firmas se incluye una variable categórica, si tiene o no departamento de I+D (Dep. I+D), y el tamaño de la empresa, medido con dos variables categóricas: mediana (51-150 trabajadores) y grande (más de 150 trabajadores) ${ }^{14}$. Esta primera variable intenta controlar por diferencias en el grado de absorción de nuevo conocimiento. La segunda, por las diferencias en la disponibilidad de recursos entre firmas más grandes y pequeñas.

Naturalmente muchos de estos determinantes relativos al diseño y la gestión son endógenos al funcionamiento del consorcio y a la elección de sus miembros. Se puede argumentar que una evaluación temprana del desempeño cambie ciertas reglas de gestión del consorcio, como, por ejemplo, la frecuencia e intensidad de comunicación entre los miembros. Además, las características de los miembros son variables de elección de los socios. Las empresas se pueden auto-seleccionar en términos de escoger socios con los cuales maximizar el impacto del instrumento. Ello implica que los resultados empíricos discutidos a con-

${ }^{14}$ El grupo de comparación, y por lo tanto excluido de las regresiones, son las empresas pequeñas (menos de 51 trabajadores). 
tinuación deben interpretarse con precaución. Éstos pueden reflejar una alta correlación entre características de los consorcios y su desempeño, pero no son estrictamente causales.

Los resultados de las estimaciones se presentan en la Tabla $\mathrm{N}^{\circ} 9$. En la columna (1) se incluyen las variables explicadas anteriormente. En las columnas siguientes se agregan otras tres variables que podrían explicar el desempeño de los consorcios. Como ha sido encontrado por Dyer et al. (2006), proyectos más ambiciosos tienden a generar mejores resultados. Para analizar esta hipótesis se incluye una variable que mide

\begin{tabular}{|c|c|c|c|c|c|}
\hline & (1) & (2) & (3) & (4) & (5) \\
\hline Mediana & $\begin{array}{c}1,004 \\
(1,93)^{*}\end{array}$ & $\begin{array}{c}1,004 \\
(2,00)^{*}\end{array}$ & $\begin{array}{c}1,032 \\
(1,96)^{*}\end{array}$ & $\begin{array}{c}1,036 \\
(2,04)^{* *}\end{array}$ & $\begin{array}{c}1,272 \\
(2,49)^{* *}\end{array}$ \\
\hline Grande & $\begin{array}{l}-0,267 \\
(0,86)\end{array}$ & $\begin{array}{l}-0,321 \\
(1,01)\end{array}$ & $\begin{array}{l}-0,235 \\
(0,77)\end{array}$ & $\begin{array}{l}-0,289 \\
(0,92)\end{array}$ & $\begin{array}{l}-0,273 \\
(0,85)\end{array}$ \\
\hline Dep, I+D & $\begin{array}{r}0,045 \\
(0,16)\end{array}$ & $\begin{array}{c}0,076 \\
(0,28)\end{array}$ & $\begin{array}{r}0,053 \\
(0,19)\end{array}$ & $\begin{array}{r}0,081 \\
(0,30)\end{array}$ & $\begin{array}{r}0,041 \\
(0,14)\end{array}$ \\
\hline Competidores & $\begin{array}{r}0,193 \\
(0,75)\end{array}$ & $\begin{array}{r}0,183 \\
(0,70)\end{array}$ & $\begin{array}{r}0,155 \\
(0,59)\end{array}$ & $\begin{array}{c}0,148 \\
(0,56)\end{array}$ & $\begin{array}{r}0,227 \\
(0,79)\end{array}$ \\
\hline Distancia & $\begin{array}{l}-0,307 \\
(1,99)^{*}\end{array}$ & $\begin{array}{l}-0,338 \\
(2,03)^{* *}\end{array}$ & $\begin{array}{l}-0,366 \\
(1,82)^{*}\end{array}$ & $\begin{array}{l}-0,394 \\
(1,85)^{*}\end{array}$ & $\begin{array}{l}-0,406 \\
(1,96)^{*}\end{array}$ \\
\hline Colaboración previa & $\begin{array}{c}0,085 \\
(0,36)\end{array}$ & $\begin{array}{c}0,058 \\
(0,25)\end{array}$ & $\begin{array}{c}0,086 \\
(0,35)\end{array}$ & $\begin{array}{c}0,059 \\
(0,24)\end{array}$ & $\begin{array}{c}0,052 \\
(0,22)\end{array}$ \\
\hline Reglas & $\begin{array}{l}-0,487 \\
(2,45)^{* *}\end{array}$ & $\begin{array}{l}-0,680 \\
(3,40)^{* * *}\end{array}$ & $\begin{array}{l}-0,547 \\
(2,53)^{* *}\end{array}$ & $\begin{array}{l}-0,735 \\
(3,34)^{* * *}\end{array}$ & $\begin{array}{l}-0,769 \\
(2,10)^{* *}\end{array}$ \\
\hline Frecuencia mensual & $\begin{array}{l}-0,717 \\
(2,86)^{* * *}\end{array}$ & $\begin{array}{l}-0,674 \\
(2,67)^{* *}\end{array}$ & $\begin{array}{l}-0,665 \\
(2,57)^{* *}\end{array}$ & $\begin{array}{l}-0,623 \\
(2,37)^{* *}\end{array}$ & $\begin{array}{l}-0,532 \\
(2,11)^{* *}\end{array}$ \\
\hline Frecuencia trimestral & $\begin{array}{l}-0,918 \\
(2,75)^{* * *}\end{array}$ & $\begin{array}{l}-1,040 \\
(2,97)^{* * *}\end{array}$ & $\begin{array}{l}-0,807 \\
(2,03)^{* *}\end{array}$ & $\begin{array}{l}-0,933 \\
(2,28)^{* *}\end{array}$ & $\begin{array}{l}-1,062 \\
(2,71)^{* * *}\end{array}$ \\
\hline Frecuencia anual & $\begin{array}{l}-0,419 \\
(0,92)\end{array}$ & $\begin{array}{l}-0,453 \\
(0,99)\end{array}$ & $\begin{array}{l}-0,400 \\
(0,96)\end{array}$ & $\begin{array}{l}-0,436 \\
(1,03)\end{array}$ & $\begin{array}{l}-0,149 \\
(0,24)\end{array}$ \\
\hline Riesgo técnico & & $\begin{array}{r}0,000 \\
(0,00)\end{array}$ & & $\begin{array}{l}-0,008 \\
(0,06)\end{array}$ & $\begin{array}{r}0,032 \\
(0,24)\end{array}$ \\
\hline Idea empresas & & & $\begin{array}{l}-0,228 \\
(0,72)\end{array}$ & $\begin{array}{l}-0,220 \\
(0,68)\end{array}$ & $\begin{array}{l}-0,095 \\
(0,32)\end{array}$ \\
\hline Apropiabilidad & & & & & $\begin{array}{l}-0,856 \\
(2,01)^{*}\end{array}$ \\
\hline Constante & $\begin{array}{c}3,603 \\
(4,45)^{* * *}\end{array}$ & $\begin{array}{c}4,125 \\
(4,43)^{* * *}\end{array}$ & $\begin{array}{l}3,983 \\
(3,54)^{* * *}\end{array}$ & $\begin{array}{l}4,494 \\
(3,59)^{* * *}\end{array}$ & $\begin{array}{c}4,411 \\
(3,15)^{* * *}\end{array}$ \\
\hline Observaciones & 56 & 55 & 56 & 55 & 54 \\
\hline R2 ajustado & 0,38 & 0,35 & 0,37 & 0,35 & 0,34 \\
\hline
\end{tabular}

Valor absoluto del estadístico $\mathrm{t}$ con errores estándares robustos entre paréntesis.

*Significativa al 10\%; ** Significativa al 5\%; *** Significativa al $1 \%$ 
el grado de riesgo técnico de los proyectos emprendidos por el consorcio. Esta variable (Riesgo) toma valores de 1 (bajo) hasta 3 (alto). En el caso chileno, la evidencia recogida de las entrevistas y la encuesta sugiere que la idea de creación del consorcio no fue impulsada fundamentalmente por las empresas. Para ver si ello tiene algún efecto sobre el desempeño se incluye una variable categórica que toma el valor 1 si la empresa señala que la idea fue impulsada por las empresas (Idea empresa) y no por otras entidades. Finalmente, se analiza si el grado de apropiabilidad de los resultados de los proyectos incide en el desempeño de los consorcios. Para ello, se construye una variable categórica que toma el valor 1 si la empresa señala que los resultados de los proyectos del consorcio podrán ser utilizados principalmente por los socios del consorcio. Se espera que una mayor apropiabilidad de los resultados debiera estar correlacionada positivamente con el desempeño.

Los resultados indican que son preferentemente las variables relativas a la gestión - frecuencia de comunicación y existencia de reglas explícitas - las que aparecen como relacionadas significativamente con el desempeño. Esta evidencia indica que la existencia de reglas explícitas y una baja frecuencia de comunicación entre los miembros tienden a asociarse con un inferior desempeño de los consorcios. En el caso de las variables de diseño, la única estadísticamente significativa es la distancia entre los miembros. Los resultados sugieren que la cercanía está asociada a un mejor desempeño. Respecto a los controles por tamaño y si la firma tiene departamento de $\mathrm{I}+\mathrm{D}$, los efectos indican que las empresas medianas evalúan su participación en los consorcios más positivamente y que no existe una relación significativa entre desempeño y la existencia de un área dedicada a la $\mathrm{I}+\mathrm{D}$ en la empresa.

En las columnas (2), (3) y (4) de la Tabla $\mathrm{N}^{\circ} 9$ se agregan variables relativas al riesgo de los proyectos y si la idea del consorcio fue impulsada por las empresas. Ambas variables resultan ser estadísticamente no significativas. Finalmente, en la columna (5) se incluye la variable que mide el grado de apropiabilidad de los resultados de los proyectos. En este caso, contrario a lo esperado, se encuentra una asociación negativa y significativa. Esto sugiere que si los resultados de los proyectos del consorcio podrán ser utilizados principalmente por las empresas participantes, ello afecta su desempeño en forma negativa. En este sentido parece ser que la mayor apropiabilidad de los resultados podría generar dificultades para las relaciones al interior del consorcio. 
Dado que existen 16 aspectos evaluados, tomar el promedio de todos ellos puede ocultar algunas diferencias importantes en términos de los factores que determinan el éxito de los consorcios en aspectos específicos. En la Tabla $\mathrm{N}^{\circ} 10$ se presentan los resultados de las estimaciones para los indicadores agrupados en tres grupos principales: innovación, acceso a conocimiento y desempeño general de las empresas.

TABLA N ${ }^{\circ} 10$ :

RESULTADOS DE LAS ESTIMACIONES POR GRUPO DE INDICADORES

\begin{tabular}{|c|c|c|c|}
\hline & Innovación & $\begin{array}{l}\text { Desempeño } \\
\text { general }\end{array}$ & $\begin{array}{l}\text { Acceso a } \\
\text { conocimiento }\end{array}$ \\
\hline Mediana & $\begin{array}{c}1,329 \\
(1,93)^{*}\end{array}$ & $\begin{array}{l}2,386 \\
(4,35)^{* * *}\end{array}$ & $\begin{array}{l}1,169 \\
(2,76)^{* * *}\end{array}$ \\
\hline Grande & $\begin{array}{l}-0,391 \\
(1,09)\end{array}$ & $\begin{array}{r}0,015 \\
(0,04)\end{array}$ & $\begin{array}{c}0,048 \\
(0,15)\end{array}$ \\
\hline Dep, $\mathrm{I}+\mathrm{D}$ & $\begin{array}{r}0,045 \\
(0,13)\end{array}$ & $\begin{array}{l}-0,776 \\
(2,62)^{* *}\end{array}$ & $\begin{array}{r}0,066 \\
(0,24)\end{array}$ \\
\hline Competidores & $\begin{array}{r}0,381 \\
(1,08)\end{array}$ & $\begin{array}{r}0,197 \\
(0,58)\end{array}$ & $\begin{array}{r}0,149 \\
(0,53)\end{array}$ \\
\hline Distancia & $\begin{array}{l}-0,612 \\
(2,33)^{* *}\end{array}$ & $\begin{array}{r}0,072 \\
(0,44)\end{array}$ & $\begin{array}{l}-0,316 \\
(1,67)\end{array}$ \\
\hline Colaboración previa & $\begin{array}{r}0,107 \\
(0,37)\end{array}$ & $\begin{array}{l}-0,384 \\
(1,26)\end{array}$ & $\begin{array}{r}0,053 \\
(0,21)\end{array}$ \\
\hline Reglas & $\begin{array}{l}-0,342 \\
(0,81)\end{array}$ & $\begin{array}{l}-0,672 \\
(1,50)\end{array}$ & $\begin{array}{l}-1,372 \\
(3,47)^{* * *}\end{array}$ \\
\hline Frecuencia mensual & $\begin{array}{l}-0,317 \\
(1,13)\end{array}$ & $\begin{array}{l}-0,579 \\
(1,50)\end{array}$ & $\begin{array}{l}-0,651 \\
(2,05)^{* *}\end{array}$ \\
\hline Frecuencia trimestral & $\begin{array}{l}-1,077 \\
(2,08)^{* *}\end{array}$ & $\begin{array}{l}-1,327 \\
(3,93)^{* * *}\end{array}$ & $\begin{array}{l}-0,816 \\
(2,08)^{* *}\end{array}$ \\
\hline Frecuencia anual & $\begin{array}{l}-0,394 \\
(0,59)\end{array}$ & $\begin{array}{r}0,745 \\
(0,73)\end{array}$ & $\begin{array}{r}0,085 \\
(0,20)\end{array}$ \\
\hline Riesgo técnico & $\begin{array}{l}-0,113 \\
(0,73)\end{array}$ & $\begin{array}{r}0,314 \\
(1,43)\end{array}$ & $\begin{array}{r}0,265 \\
(1,60)\end{array}$ \\
\hline Idea empresas & $\begin{array}{l}-0,299 \\
(0,82)\end{array}$ & $\begin{array}{c}0,526 \\
(1,36)\end{array}$ & $\begin{array}{l}-0,009 \\
(0,03)\end{array}$ \\
\hline Apropiabilidad & $\begin{array}{l}-0,794 \\
(1,67)\end{array}$ & $\begin{array}{l}-1,533 \\
(3,02)^{* * *}\end{array}$ & $\begin{array}{l}-0,895 \\
(1,85)^{*}\end{array}$ \\
\hline Constante & $\begin{array}{l}4,122 \\
(2,47)^{* *}\end{array}$ & $\begin{array}{r}1,922 \\
(1,34)\end{array}$ & $\begin{array}{l}5,279 \\
(3,83)^{* * *}\end{array}$ \\
\hline Observaciones & 53 & 47 & 53 \\
\hline R2 ajustado & 0,32 & 0,27 & 0,34 \\
\hline
\end{tabular}

Valor absoluto del estadístico t con errores estándares robustos entre paréntesis.

*Significativa al 10\%; ** Significativa al 5\%; *** Significativa al $1 \%$ 
Los resultados muestran que para los tres grupos de indicadores las empresas medianas tienden a evaluar más positivamente la contribución de los consorcios, especialmente en el desempeño general de las empresas. En este mismo caso se encuentra que empresas que tienen departamento de I+D señalan una menor contribución de los consorcios a su desempeño, pero sin efectos significativos en los indicadores de innovación y acceso al conocimiento. En el caso de la frecuencia de comunicación, consistente con el efecto encontrado para el promedio general, se aprecia que la menor frecuencia se relaciona negativamente con los tres grupos de indicadores.

Existen algunas diferencias relevantes respecto al análisis del promedio general de indicadores que vale la pena destacar. Primero, el efecto negativo de la distancia entre los miembros se da particularmente en los indicadores de innovación, pero no así en los de desempeño general y acceso a conocimiento. Segundo, la existencia de reglas afecta la evaluación de desempeño solamente respecto a los indicadores de acceso a conocimiento. Tercero, el efecto negativo de una mayor apropiabilidad de los resultados de los proyecto es significativo para los indicadores de desempeño general y acceso a conocimiento, pero no así para los de innovación.

Estos resultados revelan que la evaluación de desempeño de los consorcios y su relación con las características de los mismos es bastante compleja. Los resultados señalan que no todos los elementos de gestión y diseño afectan de igual manera a los indicadores analizados. Dos resultados, sin embargo, aparecen como robustos a través de los indicadores: las empresas medianas se favorecerían en mayor medida de la participación en los consorcios y una comunicación más frecuente entre los miembros tiende a favorecer su desempeño. Esta evidencia sería consistente con algunos resultados encontrados en otras economías, particularmente con los de Dyer et al. (2006) para Estados Unidos en el caso de la frecuencia de las comunicaciones, y con Kaiser y Kuhn (2012) respecto a que no serían las firmas de mayor tamaño las que se benefician más con su participación en consorcios. 


\section{Conclusiones}

Los resultados de las encuestas, usando un grupo amplio de indicadores, muestran que las empresas atribuyen una baja contribución de los consorcios a su desempeño. Ello es así en todos los aspectos evaluados y es consistente con la evidencia recogida en varias de las entrevistas con gerentes de consorcios y agentes de organismos públicos. En términos de los aspectos mejor evaluados, los resultados de la encuesta revelan que las tres principales contribuciones de los consorcios han sido el mejoramiento de acceso a conocimiento tecnológico, el mejoramiento de acceso a conocimiento en otras áreas (marketing, mercados internacionales, personal capacitado, etc.) y el desarrollo de proyectos tecnológicos conjuntos con empresas del consorcio. La contribución menos importante de los consorcios está asociada a la solicitud de patentes de propiedad intelectual. Dado que la mayoría de estos consorcios han estado en ejecución por sólo unos pocos años, también se indagó sobre los efectos esperados a futuro. La calificación tiende a ser generalmente más alta que la situación actual, lo que indica que potencialmente podrían contribuir a mejorar el desempeño futuro de las empresas.

Una manera de identificar las razones del bajo desempeño de los consorcios es analizar cuáles son las principales razones que las empresas señalan como obstáculos a un mejor desempeño. Los resultados de las encuestas a las empresas señalan que el principal obstáculo, aunque bajo en términos absolutos, son las diferencias de objetivos entre em-

presas y universidades y/o centros de investigación. Éste ha sido un aspecto que surge también de las entrevistas con investigadores que participan en los consorcios.

Este estudio ha analizado los factores que pueden afectar el desempeño de los consorcios. Para el desempeño general, se encuentra que las variables relativas a la gestión del consorcio tienden a ser importantes. La evidencia indica que una baja frecuencia de comunicación, la existencia de reglas y una mayor distancia entre los miembros se asocian con un desempeño inferior de los consorcios. Además, se encuentra que las empresas medianas se verían más beneficiadas por la participación en este tipo de acuerdo.

El análisis econométrico por grupos de indicadores reveló también algunos hallazgos interesantes. Los resultados señalan que no todos 
los elementos de gestión y diseño afectan de igual manera a los indicadores analizados. Dos resultados, sin embargo, aparecen como robustos a través de los indicadores: las empresas medianas se favorecerían en mayor medida de la participación en los consorcios y una comunicación más frecuente entre los miembros tiende a favorecer su desempeño.

¿Qué podemos aprender en términos de implicancias de política? Primero, dado que los resultados apuntan a un impacto relativamente bajo de estos instrumentos, esto genera dudas de si efectivamente ellos están solucionando las fallas de mercado que justifican la intervención pública. Más aun, existen aspectos importantes como la generación de innovaciones de productos y procesos, y la obtención de patentes que son aspectos pobremente evaluados por las empresas. Ello implica que los proyectos no estarían generando los efectos deseados en materia de innovación tecnológica y que, probablemente, estén cubriendo otras demandas y necesidades de las empresas.

Segundo, la percepción de los encuestados, aunque es una fuente importante de información, no está libre de sesgos y comportamientos oportunistas. Ello implica que evaluaciones más sistemáticas de estos instrumentos deban ser realizadas, antes de implementar cambios en el instrumento. Esto plantea desafíos en varias dimensiones, pero consideramos que dos son los más importantes. Primero, se debe definir qué se quiere evaluar. En este trabajo, se usan 16 aspectos, pero ninguno proviene de fuentes de información objetivas, sino más bien de percepciones de las empresas. Avanzar en este sentido requiere que los fondos asignados tengan una contraparte en resultados esperados medibles, que sean informados periódicamente por los beneficiarios. Segundo, como lo sugiere la literatura de evaluación de impacto, para determinar que tan bien o mal lo están haciendo las empresas que usan estos recursos públicos, se requiere de un contrafactual. Esto implica que no sólo este instrumento, sino varios de los utilizados por las agencias de fomento productivo y de innovación, tengan diseñada ex ante una metodología de evaluación. En estos casos, pensar en cómo obtener información de un grupo de control apropiado es fundamental.

En tal sentido, una conclusión de este estudio es que las futuras convocatorias debieran definir de manera más explícita cuáles son los resultados esperados de este instrumento. Ello permitiría definir más certeramente bajo qué criterios cuantitativos y cualitativos se debe evaluar el desempeño de los consorcios. Este trabajo se centró en variables 
de percepción de resultados del consorcio por parte de sus socios, sin embargo, es necesario estudiar a futuro los potenciales efectos de un consorcio en relación a variables observables dentro de la empresa, las cuales deben recoger en parte alguno de los objetivos del instrumento.

Aspectos más específicos en los cuales el instrumento puede ser mejorado surgen de los resultados de los determinantes del desempeño de los consorcios. La evidencia de este trabajo sugiere que aspectos relativos al diseño (distancia entre miembros) y gestión (frecuencia de comunicación y la adopción de reglas explícitas) son importantes determinantes del desempeño de los consorcios. Sin embargo, una mayor discusión de cómo estos elementos deben ser incorporados en futuros consorcios es necesaria para una optimización del instrumento.

ANEXO:

CUESTIONARIO ENCUESTA A EMPRESAS

FOLIO LIST

FOLIO DIG

\section{Proyecto Análisis de Consorcios Tecnológicos en Chile}

La Fundación para la Innovación Agraria (FIA) y la Subsecretaría de Economía han solicitado al Departamento de Economía de la Universidad de Chile, a través de su Centro Intelis, un estudio sobre Consorcios Tecnológicos en Chile. El objetivo de este trabajo es analizar el funcionamiento y desempeño de los Consorcios de manera de optimizar su impacto sobre los agentes que utilizan este instrumento.

Todos los datos y opiniones entregadas en esta encuesta se mantendrán en absoluta reserva y sujetos al secreto estadístico. La Universidad de Chile adhiere a lo establecido en La ley Orgánica $\mathrm{N}^{\circ} 17.374$ del Instituto Nacional de Estadísticas y bajo ninguna circunstancia el equipo investigador entregará información de empresas individuales a las instituciones mandantes $u$ otras personas naturales $o$ jurídicas.

\section{Características de la empresa}

Nota: Las Asociaciones Gremiales no deben contestar esta parte, y esto debe quedar en un código en la base de datos, como un "no aplica". 
a) ¿Tiene un Departamento de Investigación y Desarrollo (I+D)? 1. Sí

b) Especifique porcentaje de las ventas que fue exportado en el

$1.0 \%$ año 2008

2. $1 \%-50 \%$

3. $51-100 \%$

c) Empleo en el año 2008 (número de trabajadores contratados

1. $1-50$ de manera directa o indirecta por su empresa)

2. $51-150$

3. más de 150

d) Especifique el porcentaje del capital de la empresa que es

$1.0 \%$ de propiedad extranjera, en el año 2008

2. $1 \%-50 \%$

3. $51-100 \%$

e) ¿Ha participado con anterioridad en otros proyectos financiados 1. Sí por el sector público?

2. No

SÍ = ¿Cuál o cuáles de estos?

5.1 Profo

5.2 FAT

5.3 FDI

5.4 Fondef

5.5 Fontec

5.6 Otro Sector público (especifique)

f) Es miembro de una Asociación Gremial?

1. Sí

2. No

\section{Origen y formalización del Consorcio Tecnológico}

a) La idea de la creación del Consorcio Tec- 1. Una agencia de Gobierno nológico fue fundamentalmente impulsada 2 . Una empresa por:

3. Un grupo de empresas de la misma industria

4. Un grupo de empresas de distintas industrias

5. Una Universidad o institución de investigación

6. Una Agencia intermediaria

7. Otro (Especificar)

b) Desde la aprobación del proyecto de crea- 1. Menos de 6 meses ción del Consorcio Tecnológico hasta su 2. Entre 6 meses y un año inicio de actividades, el tiempo que pasó 3. Más de un año fue: 
c) El tiempo necesario para gestionar el inicio del Consorcio Tecnológico (Desde aprobación del proyecto de creación del Consorcio hasta el inicio de actividades) fue afectado por:

d) ¿Su empresa había desarrollado previamente algún proyecto tecnológico con otras empresas o instituciones que ahora participan en el Consorcio Tecnológico?

e) ¿Su empresa había desarrollado previamente algún proyecto tecnológico con otras empresas o instituciones que no pertenecen al Consorcio Tecnológico?
1. Poca experiencia de las empresas en el desarrollo de este tipo de proyecto conjuntos

2. Dificultades para ponerse de acuerdo entre las empresas

3. Excesiva burocracia de la agencia gubernamental

4. Otra, indique:

1. Con ninguna

2. Con algunas

3. Con la mayoría

1. Sí

2. No

\section{Características del diseño del Consorcio Tecnológico}

Indique si el Consorcio Tecnológico en que su empresa participa cuenta con las siguientes características.....(leer una a una, aplicando rotación)

a) ¿Hay en el Consorcio Tecnológico empresas de la misma 1: Sí 2: No industria que compiten directamente con su empresa?

b) ¿Hay en el Consorcio Tecnológico empresas de la misma 1: Sí 2: No industria, pero que no compiten directamente con su empresa?

c) ¿Hay en el Consorcio Tecnológico empresas de alto 1: Sí 2: No desarrollo tecnológico en comparación a su empresa?

d) ¿Hay en el Consorcio Tecnológico empresas en diferentes 1: Sí 2: No industrias?

e) ¿Hay en el Consorcio Tecnológico empresas de mayor 1: Sí 2: No tamaño en comparación a su empresa?

f) ¿Hay en el Consorcio Tecnológico vínculos formales de 1: Sí 2: No cooperación con clientes de su empresa?

g) ¿Hay en el Consorcio Tecnológico vínculos formales de 1: Sí 2: No cooperación con proveedores de insumos de su empresa?

h) ¿Hay en el Consorcio Tecnológico vínculos formales de 1: Sí 2: No cooperación con universidades o institutos públicos de investigación que no pertenecen al Consorcio? 


\section{Localización de los participantes del consorcio tecnológico}

a) Indique la distancia aproximada (en kilómetros) entre

1. $0-49 \mathrm{~km}$ su entidad y aquella entidad que participa del Consorcio Tecnológico pero se encuentra más lejana

2. $50-99 \mathrm{~km}$

3. $100-200 \mathrm{~km}$

4. más de $200 \mathrm{~km}$

b) Indique la distancia aproximada (en kilómetros) entre

1. $0-49 \mathrm{~km}$ su entidad y el coordinador (o donde se encuentra la

2. $50-99 \mathrm{~km}$ gerencia) del Consorcio Tecnológico

3. $100-200 \mathrm{~km}$

4. más de $200 \mathrm{~km}$

\section{Características de la gestión del Consorcio Tecnológico}

a) ¿El Consorcio Tecnológico cuenta con reglas explícitas

1. Sí 2. No para la salida o la entrada de empresas u otras entidades?

b) ¿El Consorcio Tecnológico cuenta con reglas explícitas para el uso de los resultados (por ejemplo, patentes e

1. Sí 2. No innovaciones de producto y procesos) de los proyectos del Consorcio?

c) Los resultados (patentes, innovaciones de productos y procesos, etc.) del Consorcio Tecnológico han sido distribuidos:

1. En partes iguales

2. De acuerdo al tamaño de los socios

3. De acuerdo a la contribución de los socios

4. No se han obtenido resultados hasta ahora

d) En promedio, las reuniones formales entre los 1. Semanal miembros del Consorcio Tecnológico ha sido de tipo: 2. Mensual

3. Trimestral

4. Anual

e) Desde el inicio del Consorcio Tecnológico, la 1. Permanecer igual frecuencia de estas reuniones ha tendido a $\quad 2$. Aumentar

3. Disminuir

f) En las decisiones estratégicas del Consorcio Tecnológico, 1: Nulo su grado de participación lo califica como: $\quad 2$ : Bajo

3: Moderadamente bajo

4: Moderadamente alto 5: Alto 


\section{Medidas de desempeño (Pasar tarjeta)}

¿En qué medida su partici- ¿En qué medida su partipación en el Consorcio cipación en el Consorcio Tecnológico ha contribuido Tecnológico espera que hasta ahora a mejorar el contribuya a futuro a desempeño de su entidad mejorar el desempeño de en los siguientes aspectos? su entidad en los siguientes aspectos?

Use la siguiente escala Use la siguiente escala
0: Nula
0: Nula
1: Baja
1: Baja
2: Moderadamente baja
2: Moderadamente baja
3: Moderadamente alta
3: Moderadamente alta
4: Alta
4: Alta

a) Introducción de productos nuevos

b) Introducción de procesos productivos nuevos

c) Introducción de diseños industriales nuevos

e) Mejoramiento de productos ya existentes

d) Mejoramiento de procesos productivos ya existentes

e) Mejoramiento de diseños industriales ya existentes

g) Patentes de propiedad intelectual solicitadas

h) Aumento de las ventas

¿En qué medida su participación en el Consorcio Tecnológico ha contribuido hasta ahora a mejorar el desempeño de su entidad en los siguientes aspectos?
¿En qué medida su participación en el Consorcio Tecnológico espera que contribuya a futuro a mejorar el desempeño de su entidad en los siguientes aspectos? 
Use la siguiente escala Use la siguiente escala

0: Nula $\quad 0:$ Nula

1: Baja 1: Baja

2: Moderadamente baja 2: Moderadamente baja

3: Moderadamente alta 3: Moderadamente alta

4: Alta 4: Alta

i) Reducción de costos de producción

j) Reducción del riesgo de la innovación

k) Mejoramiento de acceso a conocimiento tecnológico

1) Mejoramiento de acceso a conocimiento en otras áreas: marketing, mercados internacionales, personal capacitado, etc.

m) Mejoramiento en su desempeño exportador

n) Obtención de certificaciones nacionales $\mathrm{y} / \mathrm{o}$ internacionales

¿En qué medida su participación en el Consorcio Tecnológico ha contribuido hasta ahora a mejorar el desempeño de su entidad en los siguientes aspectos?

¿En qué medida su participación en el Consorcio Tecnológico espera que contribuya a futuro a mejorar el desempeño de su entidad en los siguientes aspectos?

Use la siguiente escala Use la siguiente escala
0: Nula
0: Nula
1: Baja
1: Baja
2: Moderadamente baja
2: Moderadamente baja
3: Moderadamente alta
3: Moderadamente alta
4: Alta
4: Alta

o) Desarrollo de nuevos proyectos tecnológicos conjuntos con empresas del Consorcio Tecnológico

p) Desarrollo de nuevos proyectos comerciales conjuntos con empresas del Consorcio Tecnológico 


\section{Obstáculos para el desempeño del Consorcio Tecnológico}

¿En qué medida los siguientes factores han sido un obstáculo para conseguir un mejor desempeño y gestión del Consorcio Tecnológico?

Use la siguiente escala

0 : Nula

1: Baja

2: Moderadamente baja

PASAR TARJETA

3: Moderadamente alta

4: Alta

a) La falta de recursos financieros para realizar investigación

b) La falta de infraestructura para llevar a cabo los proyectos del Consorcio Tecnológico

c) La falta de recursos humanos calificados entre los miembros del Consorcio Tecnológico

d) La falta de recursos humanos calificados en el país

e) El alto riesgo tecnológico de los proyectos emprendidos en el Consorcio Tecnológico

f) Los diferentes objetivos entre las empresas del Consorcio Tecnológico

g) Los diferentes objetivos entre las empresas y las universidades y/o centros de investigación

h) Los diferentes enfoques de investigación entre las empresas del Consorcio Tecnológico

i) Los diferentes enfoques de investigación entre las empresas y las universidades y/o centros de investigación

j) Insuficiente capacidad de las empresas para adaptar y/o absorber los desarrollos tecnológicos generados por el Consorcio Tecnológico

k) Otro obstáculo (especifique y califique usando la escala)

\section{Proyectos y modelo de negocios}

a) ¿Cuál ha sido el número de proyectos de investigación emprendidos por el Consorcio Tecnológico desde su creación?

b) ¿Cuántos trabajadores han estado trabajando directamente en los proyectos del Consorcio Tecnológico (promedio por año)?

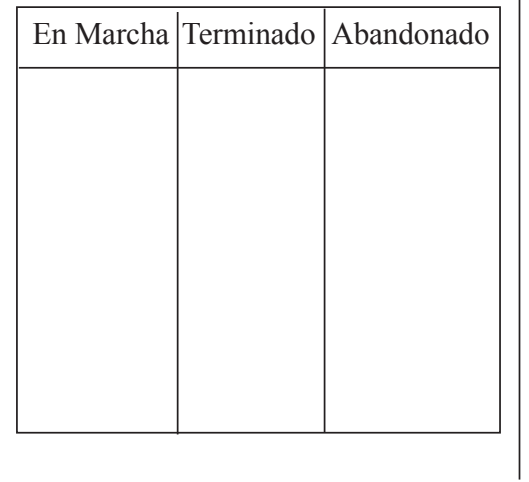


c) ¿Cuál es el riesgo técnico, a su juicio,

2. Bajo de los proyectos emprendidos por el

3. Medio

Consorcio Tecnológico?

4. Alto

d) ¿Cuál es el riesgo comercial, a su juicio, de los proyectos emprendidos por el Consorcio Tecnológico?
a. Bajo
b. Medio
c. Alto

e) Al inicio de Consorcio Tecnológico, ¿En cuánto se estimaba en su empresa el plazo promedio para obtener

1. Dentro de 1 año

2. Dentro de 5 años resultados sustantivos de los proyectos de investigación ejecutados por el Consorcio Tecnológico?

f) ¿Cree que los resultados de los proyectos del Consorcio Tecnológico podrán ser utilizados principalmente por:

3. Dentro de 10 años

4. En más de 10 años

1. Sólo los socios del consorcio

2. Todas las empresas de la industria

3. Empresas de la industria, así como también por proveedores y clientes

4. Empresas de otros sectores económicos

g) El objetivo principal de los proyectos de investigación del Consorcio Tecnológico es:

II. Lograr el cumplimiento de certificaciones nacionales o internacionales

III. Reducir costos de producción

IV. Reducir riesgos de producción

V. Acceder a nuevos mercados

VI. Desarrollo de nuevos productos

VII. Mejoramiento de productos ya existentes

VIII. Nuevos procesos productivos

IX. Mejoramiento de procesos productivos ya existentes

X. ¿Hay algún otro? .... ¿Cuál?:

\section{Vinculación con agencias gubernamentales}

¿Cuán importante ha sido el rol de la agencia pública en:

Use la siguiente escala

0 : Nula

1: Baja

PASAR TARJETA

2: Moderadamente baja

3: Moderadamente alta

4: Alta 

a) El origen de la idea para formar el Consorcio Tecnológico
b) La puesta en marcha de las actividades del Consorcio Tecnológico
c) Facilitar la interacción con los miembros del Consorcio Tecnológico
d) Apoyo cuando ha habido problemas financieros en el Consorcio Tecnológico
e) Apoyo cuando ha habido problemas de gestión en el Consorcio Tecnológico
f) Apoyo con la información relevante para la solución de problemas tecnológicos
g) Apoyo en la evaluación de los resultados
h) Apoyo en la entrega de recomendaciones oportunas y relevantes para el cumplimiento de los objetivos

\section{REFERENCIAS}

Acosta, J. y A. Modrego (2001). "Public Financing of Cooperative R\&D Projects in Spain: The Concerted Projects under the National R\&D Plan". Research Policy, 30 (4): 625-641.

Álvarez, R., J. M. Benavente, C. Contreras y J. L. Contreras (2012). "Consorcios Tecnológicos en Argentina, Chile, Colombia y Uruguay". El Trimestre Económico, LXXIX (1): 227-256.

Allen Consulting Group (2005). "The Economic Impact of Cooperative Research Centres in Australia”. Report for the Cooperative Research Centres Association Inc.

Almeida, P. y B. Kogut (1999). "Localization of Knowledge and the Mobility of Engineers in Regional Networks". Management Science, 45 (7): 905912 .

Branstetter y Sakakibara (1998). “Japanese Research Consortia: A Microeconometric Analysis of Industrial Policy". Journal of Industrial Economics XLVI (2): 207-233. (2002). "When do Research Consortia Work Well and Why? Evidence from Japanese Panel Data". American Economic Review 92 (1): 143-159.

Casalet, M. y F. Stezano (2006). “Cambios Institucionales para la Innovación: Nuevos Instrumentos de Política Científica y Tecnológica. El caso del Consorcio Xignux-CONACYT". Mimeo.

Czarnitzky, D. y A. Fier (2003). "Publicly Funded R\&D Collaborations and Patent Outcome in Germany". ZEW Discussion Paper N 03-24.

Dyer, J. H., B. C. Powell, M. Sakakibara y A. J. Wang (2006). "Determinants of Success in R\&D Alliances". National Institute of Standards and Technology.

Irwin, D. y P. Klenow (1996). "High-Tech R\&D Subsidies: Estimating the Effects of Sematech". Journal of International Economics 40: 323-344. 
Kaiser, U. y J. M. Kuhn (2012): “Long-Run Effects of Public-Private Research Joint Ventures: The Case of the Danish Innovation Consortia Support Scheme". Research Policy, 41(5): 913-927.

Katz, M. L. (1986). "An Analysis of Cooperative Research and Development”. RAND Journal of Economics, 17 (4): 527-543.

Sakakibara, M. (2001). "The Diversity of R\&D Consortia and Firm Behavior: Evidence from Japanese Data”. Journal of Industrial Economics, 49 (2): 181-196.

Saxeniam A. (1994). "Regional Advantage: Culture and Competition in Silicon Valley and Route 128". Harvard University Press: Cambridge, MA. 